\title{
The pion distribution amplitude and the pion-photon transition form factor in a nonlocal chiral quark model
}

\author{
D. Gómez Dumm ${ }^{a, b}$, S. Noguera ${ }^{c}$, N. N. Scoccola ${ }^{b, d, e}$ and S. Scopetta ${ }^{f}$ \\ ${ }^{a}$ IFLP, CONICET - Dpto. de Física, \\ Universidad Nacional de La Plata, C.C. 67, (1900) La Plata, Argentina \\ ${ }^{b}$ CONICET, Rivadavia 1917, (1033) Buenos Aires, Argentina \\ ${ }^{c}$ Departamento de Física Teórica and Instituto de Física Corpuscular, \\ Universidad de Valencia-CSIC, E-46100 Burjassot (Valencia), Spain \\ ${ }^{d}$ Physics Department, Comisión Nacional de Energía Atómica, \\ Av. Libertador 8250, (1429) Buenos Aires, Argentina \\ e Universidad Favaloro, Solís 453, (1078) Buenos Aires, Argentina and \\ ${ }^{f}$ Dipartimento di Fisica, Università di Perugia, and INFN, \\ Sezione di Perugia, via A. Pascoli, I-06100 Perugia, Italy
}

\begin{abstract}
We study the pion Distribution Amplitude $(\pi \mathrm{DA})$ in the context of a nonlocal chiral quark model. The corresponding Lagrangian reproduces the phenomenological values of the pion mass and decay constant, as well as the momentum dependence of the quark propagator obtained in lattice calculations. It is found that the obtained $\pi \mathrm{DA}$ has two symmetric maxima, which arise from the new contributions generated by the nonlocal character of the interactions. This $\pi \mathrm{DA}$ is applied to leading order and next-to-leading order calculations of the pion-photon transition form factor. Implications of the results are discussed.
\end{abstract}




\section{INTRODUCTION}

The pion Distribution Amplitude $(\pi \mathrm{DA})$ is a fundamental theoretical ingredient in the description of exclusive high-energy processes. The simplest hard exclusive process determined by the $\pi \mathrm{DA}$ is the transition $\pi \rightarrow \gamma \gamma^{*}$ at high photon virtuality $Q^{2}$, since for this process the pion is the only hadron involved; on the other hand, the large $Q^{2}$ behavior of the related Pion Transition Form Factor ( $\pi$ TFF) is well known from perturbative QCD $[1,2]$. The $\pi$ TFF can be measured for both space-like and time-like momentum transfers through the processes $e^{+} e^{-} \rightarrow e^{+} e^{-} \pi^{0}$ and $e^{+} e^{-} \rightarrow \pi^{0} \gamma$, respectively. The corresponding experimental status has been improved in the last years, since old results from CELLO [3] (covering a space-like momentum transfer region $\left.0.68<Q^{2}<2.17 \mathrm{GeV}^{2}\right)$ and CLEO [4] $\left(1.64<Q^{2}<7.9 \mathrm{GeV}^{2}\right)$ have now been complemented with data from the BABAR [5] and BELLE [6] Collaborations, which cover pion virtualities ranging from 4 to $35 \mathrm{GeV}^{2}$. While the old data suggested that the $\pi \mathrm{TFF}$ reaches its asymptotic behavior for $Q^{2}$ values of the order of a few $\mathrm{GeV}^{2}$, the new BABAR data exhibit a steeper growth, indicating that the asymptotic QCD limit is crossed at $Q^{2} \sim 10 \mathrm{GeV}^{2}$. The BELLE data show instead a slower growth, in which the $\pi$ TFF seems to cross the asymptotic limit at $Q^{2} \sim 20 \mathrm{GeV}^{2}$. In addition, the BABAR Collaboration has recently measured the $\eta$ and $\eta^{\prime}$ transition form factors [7]; the data show in this case a mild behavior, approaching from below the corresponding asymptotic QCD limit for large $Q^{2}$ values. In any case, owing to the relatively large errors, it could be said that present experimental data are compatible with each other, and still more accurate measurements would be needed in order to firmly establish the behavior of the $\pi \mathrm{TFF}$ in the region of intermediate and large $Q^{2}$.

In view of the new experimental results, a significant theoretical effort has been carried out towards the obtention of theoretical predictions for the $\pi$ DA and $\pi$ TFF. First analyses have proposed a flat $\pi$ DA, i.e. $\phi_{\pi}(x)=1[8,9]$. This scenario is compatible with QCD sum rules [10] and lattice QCD results [11, 12], which lead to values for the second moment of the $\pi$ DA that are large in comparison with that obtained using the asymptotic $\pi$ DA $\phi_{\pi}(x)=6 x(1-x)$. A constant $\pi \mathrm{DA}$ is in fact obtained within effective theories such as the Nambu-Jona-Lasinio (NJL) model [13-15] and the "spectral" quark model [16]. A formalism which connects the experimental parametrization of the $\pi \mathrm{TFF}$ at low photon virtuality with the description of the $\pi \mathrm{TFF}$ at high photon virtuality using a flat $\pi$ DA has been devel- 
oped in Ref. [17]. Within this formalism, a good agreement with the experimental pattern is achieved after the inclusion of a correction carrying an extra power of $1 / Q^{2}$, which is needed in order to reproduce the data in the region $1<Q^{2}<15 \mathrm{GeV}^{2}$. With the same ingredients, in the context of the NJL model a good description of the $\eta$ TFF can be obtained [18]. Finally, other analyses carried out within quark models can be found in Refs. [19-23]. Another recent calculation of the $\pi$ DA has been performed within the AdS/QCD holographic correspondence hypothesis [24]. The $\pi \mathrm{TFF}$ obtained by this approach cannot explain the trend of the recent BABAR data [25]. The same problem is encountered also in the analysis of Ref. [26]. The $\pi$ DA has been recently evaluated also within the Dyson-Schwinger equation framework [27]; first lattice estimates of this quantity have been presented in Ref. [28]. In all these approaches the $\pi$ DA is obtained through theoretical calculations, and parameters are fitted from other physical quantities.

The $\pi$ DA and the $\pi$ TFF have also been studied within the Non Local Condensates Sum Rule (NLC-SR) and Light Cone Sum Rule (LCSR) approaches [29-34]. These calculations use similar ingredients, introducing corrections with extra powers of $1 / Q^{2}$ in order to describe the data through the twist 4 and 6 contributions. While in Refs. [33, 34] a good description of the experimental results is obtained, in Refs. [32] it is claimed that in order to reproduce the data from BABAR one would need some enhancement mechanism that cannot be explained within the standard QCD scheme based on collinear factorization. A study of the $\pi$ DA is also presented in Refs. [35, 36], starting from the pion leading twist wave function. In general, in all these works the $\pi \mathrm{DA}$ is parametrized in terms of an expansion in a series of Gegenbauer polynomials. This expansion is truncated keeping the first few polynomials, and the corresponding coefficients are treated as parameters to be adjusted.

The aim of this work is to study the $\pi \mathrm{DA}$ and the $\pi \mathrm{TFF}$ within the framework of a nonlocal Nambu-Jona-Lasinio model (nlNJL). The NJL model is a simple scheme based on the QCD feature of dynamical chiral symmetry breaking, in which quarks interact through a local, chiral invariant four-fermion coupling. The local nature of this interaction allows to obtain simple solutions of the corresponding Dyson-Schwinger and Bethe-Salpeter equations. However, the main drawbacks of the model are direct consequences of the locality: a definite prescription is needed in order to regularize ultraviolet loop divergences, and the model is nonconfining. The nlNJL model represents an improvement over the local theory. Indeed, it can be seen that nonlocal form factors regularize the model in such a way that anomalies 
are preserved and charges are properly quantized, and there is no need to introduce extra cut-offs. In fact, nonlocality arises naturally in quantum field theory when the interactions involve large coupling constants.

The starting point in our analysis will be a Lagrangian theory that includes couplings between nonlocal quark currents. In this way, our formalism ensures the preservation of fundamental symmetries (chiral, Poincaré and local electromagnetic gauge invariances) that guarantee the proper normalization of the $\pi \mathrm{DA}$. In the framework of a Lagrangian theory, the three main ingredients of a nonperturbative analysis that involves photons and the pion are: i) the quark propagator, which obeys the Dyson-Schwinger equation; ii) the description of the pion as a bound state of a Bethe-Salpeter equation (BSE); iii) a prescription for introducing the electroweak interaction that preserves gauge symmetry. Owing to the chiral symmetry, the kernels of the equations appearing in $i$ ) and $i i$ ) are not independent [37]. The Dyson-Schwinger equation leads to momentum dependences in the quark propagators through its mass and its wave function renormalization. In our scheme the gluons have been integrated out (we have only flavor interaction between quarks), and confinement is obtained from the structure of the quark propagator and by limiting the Fock space to color singlet states. The pion is described in a consistent way by solving the BSE, and it shows up as a Goldstone boson associated with the spontaneous breakdown of the chiral symmetry. Finally, the couplings involving photons and weak bosons are implemented by imposing local gauge invariance in the Lagrangian. Therefore, we must gauge not only the kinetic term, but also the nonlocal quark currents in the interaction terms.

The quark propagator is taken as one of the main ingredients of our model. The reason is that one has direct information on this propagator from the fundamental QCD theory, since the momentum dependences of quark mass and wave function renormalization have been calculated in lattice QCD $[38,39]$. Our Lagrangian is in fact the minimal framework that allows to incorporate the full momentum dependence obtained through these lattice calculations. In this way, our model can be seen as an extension of nonlocal NJL models analyzed in previous works [19, 40-44], but with a particular philosophy. The model considered here has been proposed in Ref. [45], and then it has been successfully applied to the analysis of different hadronic observables [46-48].

Once the Lagrangian theory has been defined, it is possible to obtain the $\pi$ DA from a fundamental calculation. The main difficulty to be solved is that the bilocal axial current 
present in the definition of the $\pi \mathrm{DA}$ will be dressed by the nonlocal interaction. To deal with this problem we rely on the basic physical idea beyond the factorization of the $\pi$ TFF into hard and soft contributions for high $Q^{2}$ : the struck quark loses its high momentum before being able to interact with the remaining quarks and gluons of the hadron. This situation will be implemented here by considering the bilocal current associated to the $\pi$ DA as a current coupled to an external fictitious probe carrying the adequate quantum numbers.

The $\pi$ DA provides the dominant twist two contribution to the $\pi$ TFF. Corrections to this term will be introduced considering contributions that carry extra powers of $1 / Q^{2}$ (we will include $1 / Q^{4}$ and $1 / Q^{6}$ terms). Therefore, in our scheme we have a fixed $\pi \mathrm{DA}$ and two free parameters in the $\pi \mathrm{TFF}$. This is in contrast with the program followed in Refs. [30-36], where the $\pi \mathrm{DA}$ is parametrized in terms of a expansion in Gegenbauer polynomials with free coefficients and the twist four and six corrections are constrained by sum rule techniques.

The present paper is organized as follows. In section II we describe the connection between the $\pi \mathrm{TFF}$ and the $\pi \mathrm{DA}$, we present the model Lagrangian and we quote our analytical results for the $\pi$ DA. In section III we show and discuss the numerical results for the $\pi$ DA obtained within our model. The dependence on the transverse momentum $k_{T}$ and the connection with the light cone wave functions are discussed in section IV. In section $\mathrm{V}$ the results obtained for the $\pi \mathrm{TFF}$ are analyzed. Finally, in section VI we sketch our conclusions. Details of the calculations, including some relevant analytical expressions, can be found in Appendices A and B.

\section{FORMALISM}

\section{A. Generalities on the evaluation of the $\pi \mathrm{TFF}$ and $\pi \mathrm{DA}$ in effective quark models}

At stated, the transition form factor for the process $\pi^{0} \rightarrow \gamma \gamma^{*}$ at large photon virtuality is basically determined by the pion distribution amplitude. At the leading order in powers of $1 / Q^{2}$ one has

$$
F\left(Q^{2}\right)=\frac{\sqrt{2} f_{\pi}}{3 Q^{2}} \int_{0}^{1} d x T_{H}\left(x, Q^{2}, \mu\right) \phi_{\pi}(x, \mu),
$$

where $f_{\pi}=0.131 \mathrm{GeV}$. Here the function $T_{H}\left(x, Q^{2}, \mu\right)$, which includes both photon vertices (see Fig. 1), accounts for the hard contributions to the process and can be calculated from perturbative QCD. In the modified minimal subtraction $(\overline{\mathrm{MS}})$ scheme, up to the NLO in 


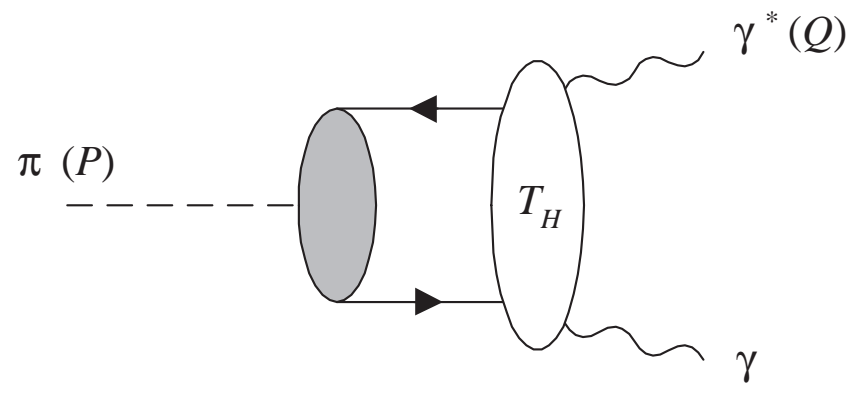

FIG. 1: Schematic structure of the QCD factorization for the $\pi \rightarrow \gamma \gamma^{*}$ process.

the strong coupling, one obtains $[49,50]$

$$
T_{H}^{\mathrm{NLO}}\left(x, Q^{2}, \mu\right)=\frac{1}{x}\left\{1+C_{F} \frac{\alpha_{\mathrm{s}}(\mu)}{4 \pi}\left[\ln ^{2} x-\frac{x \ln x}{1-x}-9+(3+2 \ln x) \ln \frac{Q^{2}}{\mu^{2}}\right]\right\},
$$

with $C_{F}=4 / 3$ for $N_{c}=3$. On the other hand, $\phi_{\pi}(x, \mu)$ stands for the $\pi \mathrm{DA}$, which involves the soft, nonperturbative contributions to the form factor [in Eq. (2), the symmetry property $\phi_{\pi}(1-x, \mu)=\phi_{\pi}(x, \mu)$ has been used]. One can take this distribution amplitude from some theoretical model for the pion or, alternatively, it can be parametrized phenomenologically. Finally, the parameter $\mu$ is the renormalization and factorization scale, which will be set here by $\mu^{2}=Q^{2}$. Different relations between $\mu^{2}$ and $Q^{2}$ have been considered in Ref. [33]. In fact, our results do not show a significant numerical variation for these different choices. For simplicity, in the following we will omit the $\mu$ dependence in $\phi_{\pi}(x, \mu)$ unless necessary.

We will postpone the analysis of the $\pi$ TFF to Sec. V, and concentrate now in the evaluation of the $\pi$ DA within the framework of an effective quark model. By definition, the $\pi \mathrm{DA}$ $\phi(x)$ is given by

$$
i \sqrt{2} f_{\pi} \phi_{\pi}(x)=\left.\int \frac{d z^{-}}{2 \pi} e^{i P^{+} z^{-}\left(x-\frac{1}{2}\right)}\left\langle 0\left|\bar{\psi}\left(-\frac{z}{2}\right) \gamma^{+} \gamma_{5} \tau^{-} \psi\left(\frac{z}{2}\right)\right| \pi^{-}(P)\right\rangle\right|_{z^{+}=0, \vec{z}_{T}=0},
$$

where we have introduced the light front components $P^{+}, z^{-}, \gamma^{+}$, choosing a frame in which $\vec{P}_{T}=0$. For any four-vector $a^{\mu}$, the light front components are defined by $a^{ \pm}=\left(a^{0} \pm a^{3}\right) / \sqrt{2}$, while $\vec{a}_{T} \equiv\left(a^{1}, a^{2}\right)$. As it is well known, $x$ becomes the fraction of the + component of the momentum carried by the struck quark in the meson, and its support is the interval $[0,1]$.

Recalling that the pion decay constant can be defined by

$$
f_{\pi}=\frac{1}{i \sqrt{2} P^{+}}\left\langle 0\left|\bar{\psi}(0) \gamma^{+} \gamma_{5} \tau^{-} \psi(0)\right| \pi^{-}(P)\right\rangle,
$$


from Eq. (3) one obtains for $\phi_{\pi}(x)$ the sum rule

$$
\int_{0}^{1} d x \phi_{\pi}(x)=1 .
$$

It is worth stressing that this is not a normalization condition to be imposed, but a result that has to be fulfilled by any well defined model.

\section{B. $\pi$ DA in a nonlocal NJL model with wavefunction renormalization}

We consider here a nonlocal covariant $\mathrm{SU}(2)$ chiral quark model that includes wave function renormalization in the quark propagator. The corresponding Euclidean action reads $[45,47]$

$$
S_{E}=\int d^{4} y\left\{\bar{\psi}(y)\left(-i \not \partial+m_{c}\right) \psi(y)-\frac{G_{S}}{2}\left[j_{a}(y) j_{a}(y)+j_{P}(y) j_{P}(y)\right]\right\} .
$$

Here $m_{c}$ is the current quark mass, which is assumed to be the same for $u$ and $d$ quarks, while the nonlocal currents $j_{a}(y), j_{P}(y)$ are given by

$$
\begin{aligned}
& j_{a}(y)=\int d^{4} z \mathcal{G}(z) \bar{\psi}\left(y+\frac{z}{2}\right) \Gamma_{a} \psi\left(y-\frac{z}{2}\right), \\
& j_{P}(y)=\int d^{4} z \mathcal{F}(z) \bar{\psi}\left(y+\frac{z}{2}\right) \frac{i \overleftrightarrow{\phi}}{2 \varkappa_{p}} \psi\left(y-\frac{z}{2}\right)
\end{aligned}
$$

where $\Gamma_{a}=\left(\mathbb{1}, i \gamma_{5} \vec{\tau}\right)$ and $u\left(y^{\prime}\right) \overleftrightarrow{\partial} v(y)=u\left(y^{\prime}\right) \partial_{y} v(y)-\partial_{y^{\prime}} u\left(y^{\prime}\right) v(y)$. The nonlocal character of the interactions is provided by the covariant vertex form factors $\mathcal{G}(z)$ and $\mathcal{F}(z)$ in Eq. (7). In the mean field approximation these functions determine the momentum dependence of the mass and wave function renormalization in the quark propagator,

$$
\mathcal{D}_{0}(p)^{-1}=\frac{z_{p}}{-p^{\prime}+m_{p}}
$$

where

$$
z_{p} \equiv z(p)=\left(1-\bar{\sigma}_{2} f_{p}\right)^{-1}, \quad m_{p} \equiv m(p)=z_{p}\left(m_{c}+\bar{\sigma}_{1} g_{p}\right)
$$

The functions $g_{p}$ and $f_{p}$ in these equations are the Fourier transforms of $\mathcal{G}(z)$ and $\mathcal{F}(z)$, while $\bar{\sigma}_{1,2}$ are the mean field values of the scalar fields associated with the currents $j_{0}(y)$ and $j_{P}(y)$, respectively. The main point here is that starting from a given expression for $m_{p}$ and $z_{p}$, based in our case on lattice results, we can use Eq. (9) for extracting the related $g_{p}$ 
and $f_{p}$ functions. The mean field values $\bar{\sigma}_{1,2}$ are related to the values of $m_{p}$ and $z_{p}$ at $p=0$ through

$$
\bar{\sigma}_{2}=1-\frac{1}{z(0)}, \quad \bar{\sigma}_{1}=\frac{m(0)}{z(0)}-m_{c} .
$$

Following Ref. [47], we choose $m_{p}$ and $z_{p}$ as

$$
\begin{aligned}
& m_{p}=m_{c}+\frac{\alpha_{m}}{1+\left(p^{2} / \Lambda_{0}^{2}\right)^{3 / 2}}, \\
& z_{p}=1+\frac{\alpha_{z}}{\left[1+\left(p^{2} / \Lambda_{1}^{2}\right)\right]^{5 / 2}},
\end{aligned}
$$

where $m_{c}=2.37 \mathrm{MeV}, \alpha_{m}=309 \mathrm{MeV}, \alpha_{z}=-0.3, \Lambda_{0}=850 \mathrm{MeV}$ and $\Lambda_{1}=1400 \mathrm{MeV}$. This parametrization allows to reproduce very well the momentum dependence of the quark propagator mass and wave function renormalization obtained in lattice calculations [38, 39], providing at the same time the proper physical values for the pion mass and decay constant [47].

Given this effective model for the strong interactions at low energies, one can explicitly evaluate the $\pi$ DA from Eq. (3). Since the amplitude involves a bilocal axial vector current, one should introduce into the effective action in Eq. (6) a coupling to an external axial gauge field $a_{\mu}$. For a local theory this can be done by performing the replacement

$$
\partial_{\mu} \rightarrow \partial_{\mu}+i \mathcal{A}_{\mu}(y)
$$

where, according to the quantum numbers of the $\pi^{-}$field,

$$
\mathcal{A}_{\mu}(y)=\tau^{-} \gamma_{5} a_{\mu}(y)
$$

In the case of the above described nlNJL model the situation is more complicated since the inclusion of gauge interactions implies a change not only in the kinetic terms in the Lagrangian but also in the nonlocal currents appearing in the interaction terms. If $y$ and $z$ denote the space variables in the definitions of the nonlocal currents [see Eq. (7)], one has

$$
\begin{aligned}
\psi(y-z / 2) & \rightarrow W(y, y-z / 2) \psi(y-z / 2), \\
\psi^{\dagger}(y+z / 2) & \rightarrow \psi^{\dagger}(y+z / 2) W(y+z / 2, y) .
\end{aligned}
$$

Here the function $W(s, t)$ is defined by

$$
W(s, t)=\mathrm{P} \exp \left[i \int_{s}^{t} d r_{\mu} \mathcal{A}_{\mu}(r)\right],
$$


where $r$ runs over an arbitrary path connecting $s$ with $t$.

This procedure has been analyzed in detail within nlNJL models, in particular regarding the calculation of the pion decay constant [40, 41, 45], see Eq. (4). The situation is similar for the case of the bilocal axial current in the definition of the $\pi$ DA. In fact, the basic physical idea beyond the factorization of the $\pi \mathrm{TFF}$ into hard and soft contributions is that for high $Q^{2}$ the struck quark loses its high momentum before being able to interact with the remaining quarks and gluons of the hadron $\left(Q^{2} \sim 1 \mathrm{GeV}^{2}\right.$ implies a time scale of the order of $10^{-24} \mathrm{~s}$ ). Therefore, the nonlocal interaction does not see the struck quark but only the quarks in the hadron before and after the photon absorption-emission process. This can be effectively implemented by introducing an external fictitious probe carrying the adequate quantum numbers, which in our case is an axial gauge field (a similar situation has been studied in the case of the pion Parton Distribution, see Refs. [45, 46]). Thus, the axial vertex in Eq. (3) will become dressed by the nonlocal interaction, irrespective of whether the quark current is a local or a bilocal one (as in this case).

The steps to be followed in the explicit calculation of the $\pi \mathrm{DA}$ within the nlNJL model are detailed in Appendix A. We quote here the resulting expression

$$
\phi_{\pi}(x)=\frac{2 \sqrt{2} N_{c} g_{\pi q \bar{q}}}{f_{\pi}} \int \frac{d w d^{2} k_{T}}{(2 \pi)^{4}} F\left(w, x, k_{T}\right),
$$

where $g_{\pi q \bar{q}}$ stands for an effective quark-meson coupling constant (see Appendix A). It is convenient to separate the integrand in Eq. (16) into two pieces,

$$
F\left(w, x, k_{T}\right)=F_{1}\left(w, x, k_{T}\right)+F_{2}\left(w, x, k_{T}\right) .
$$

The explicit expressions for these functions are

$$
\begin{gathered}
F_{1}\left(w, x, k_{T}\right)=\frac{g_{k}}{2} \frac{z_{k_{+}} z_{k_{-}}}{D_{k_{+}} D_{k_{-}}}\left(\frac{1}{z_{k_{+}}}+\frac{1}{z_{k_{-}}}\right)\left[(1-x) m_{k_{+}}+x m_{k_{-}}\right], \\
F_{2}\left(w, x, k_{T}\right)=g_{k} \frac{z_{k_{+}} z_{k_{-}}}{D_{k_{+}} D_{k_{-}}}\left\{\left[k_{+} \cdot k_{-}+m_{k_{+}} m_{k_{-}}\right] \nu_{1}-\right. \\
\left.k \cdot\left[k_{+} m_{k_{-}}-k_{-} m_{k_{+}}\right] \nu_{2}\right\}-\frac{m_{k} z_{k}}{D_{k} \bar{\sigma}_{1}} \nu_{1},
\end{gathered}
$$

where we have defined $k_{ \pm}=k \pm P / 2$ and $D_{k}=k^{2}+m_{k}^{2}$. In terms of the variables $w$ and $k_{T}$ we have

$$
k^{2}=-i w\left(x-\frac{1}{2}\right)+m_{\pi}^{2}\left(x-\frac{1}{2}\right)^{2}+k_{T}^{2}, \quad k \cdot P=-i \frac{w}{2}
$$


Finally, the functions $\nu_{1}$ and $\nu_{2}$ in Eq. (19) are given by

$$
\begin{aligned}
& \nu_{1}=\frac{\left(x-\frac{1}{2}\right)}{k \cdot P}\left[\frac{m_{k_{+}}}{z_{k_{+}}}+\frac{m_{k_{-}}}{z_{k_{-}}}-2 \frac{m_{k}}{z_{k}}+m_{\pi}^{2} \bar{\sigma}_{1} \alpha_{g}^{-}\right]+\bar{\sigma}_{1} \alpha_{g}^{-}, \\
& \nu_{2}=\frac{\left(x-\frac{1}{2}\right)}{k \cdot P}\left[\frac{1}{z_{k_{-}}}-\frac{1}{z_{k_{+}}}+m_{\pi}^{2} \bar{\sigma}_{2} \alpha_{f}^{+}\right]+\bar{\sigma}_{2} \alpha_{f}^{+},
\end{aligned}
$$

where $\alpha_{g}^{-}$and $\alpha_{f}^{+}$depend in general on the integration path in Eq. (15). If one takes a straight line path the corresponding explicit expressions read

$$
\alpha_{g}^{-}=\int_{0}^{1} d \lambda \frac{\lambda}{2} g_{k-\lambda P / 2}^{\prime}-\int_{-1}^{0} d \lambda \frac{\lambda}{2} g_{k-\lambda P / 2}^{\prime}, \quad \alpha_{f}^{+}=\int_{-1}^{1} d \lambda \frac{\lambda}{2} f_{k-\lambda P / 2}^{\prime} .
$$

\section{LO and NLO evolution of the $\pi$ DA}

Once the $\pi \mathrm{DA} \phi(x)$ is known at a given $\mu_{0}$ scale, its evolution up to a new scale $\mu$ can be obtained from perturbative QCD $[1,2]$. In order to calculate this evolution (we denote now explicitly the $\mu$ dependence of the $\pi \mathrm{DA})$, it is convenient to expand $\phi_{\pi}(x, \mu)$ in a series of Gegenbauer polynomials,

$$
\phi_{\pi}(x, \mu)=6 x(1-x) \sum_{n=0 \text { (even) }}^{\infty} a_{n}(\mu) C_{n}^{3 / 2}(2 x-1) .
$$

From the orthogonality relations satisfied by these polynomials one gets the coefficients at the $\mu_{0}$ scale, namely

$$
a_{n}\left(\mu_{0}\right)=\frac{2(2 n+3)}{3(n+1)(n+2)} \int_{0}^{1} d x C_{n}^{3 / 2}(2 x-1) \phi_{\pi}\left(x, \mu_{0}\right) .
$$

If $\phi_{\pi}\left(x, \mu_{0}\right)$ satisfies the sum rule Eq. (5), then the first coefficient $a_{0}\left(\mu_{0}\right)$ has to be equal to 1 . Thus all the information from the pion effective model is included in the remaining coefficients $a_{n}\left(\mu_{0}\right)$, with $n=2,4, \ldots$. At the LO in the strong coupling $\alpha_{s}$ the coefficients turn out to be renormalized multiplicatively,

$$
a_{n}^{\mathrm{LO}}(\mu)=a_{n}\left(\mu_{0}\right) E_{n}^{\mathrm{LO}}\left(\mu, \mu_{0}\right)
$$

whereas at the NLO the evolution equations for different coefficients get mixed, and the pattern becomes more complicated. One has [33]

$$
a_{n}^{\mathrm{NLO}}(\mu)=a_{n}\left(\mu_{0}\right) E_{n}^{\mathrm{NLO}}\left(\mu, \mu_{0}\right)+\frac{\alpha_{s}(\mu)}{4 \pi} \sum_{k=0 \text { (even) }}^{n-2} a_{k}\left(\mu_{0}\right) E_{k}^{\mathrm{LO}}\left(\mu, \mu_{0}\right) d_{n}^{k}\left(\mu, \mu_{0}\right)
$$


Explicit expressions for the renormalization factors $E_{n}^{\mathrm{LO}}\left(\mu, \mu_{0}\right), E_{n}^{\mathrm{NLO}}\left(\mu, \mu_{0}\right)$, as well as for the off-diagonal mixing coefficients $d_{n}^{k}\left(\mu, \mu_{0}\right)$ in the $\overline{\mathrm{MS}}$ scheme, are collected in Appendix B. Usually the calculation of a few coefficients $a_{n}(\mu)$ is sufficient to get a good estimate of the $\pi$ DA at the scale $\mu$ using Eq. (21).

\section{PION DISTRIBUTION AMPLITUDE}

Our result for the $\pi \mathrm{DA}$, Eq. (16), is plotted in Fig. 2 (solid line), where the contributions coming from Eqs. (18) and (19) are also separately shown (dashed and dotted lines, respectively). One observes that the full result has two symmetric maxima. This feature is also shown by the $\pi$ DA calculated in Refs. $[10,29]$, but in our case the two maxima are much closer to $x=0.5$. From the curves it is seen that this shape arises from the term in Eq. (19), which is a genuine nonlocal contribution.

Our calculation is performed in Euclidean space. To check the consistency of our scheme we rely on the verification of the following fundamental properties of the $\pi \mathrm{DA}$ : (i) the $\pi \mathrm{DA}$ has to be invariant under the exchange $x \leftrightarrow(1-x)$; (ii) the $\pi$ DA has support in the interval $[0,1]$; (iii) the sum rule Eq. (5) has to be fulfilled.

The first property is a consequence of isospin symmetry. It can be easily checked from the analytical expressions in Eqs. (16-20).

Concerning the second property, we notice that it can be associated to the Wick rotation in cases where an exact solution can be obtained [17]. Let us assume that quark masses do not depend on the momentum, and let us write the denominators $D_{k^{ \pm}}$in Eqs. (18-19) in Minkowski space:

$$
\begin{aligned}
& D_{k^{-}}=\left(k_{-}^{2}-m^{2}+i \epsilon\right)=-2 P^{+}(1-x)\left[\left(k^{-}-\frac{P^{-}}{2}\right)+\frac{\vec{k}_{T}^{2}+m^{2}}{2 P^{+}(1-x)}-\frac{i \epsilon}{2 P^{+}(1-x)}\right] \\
& D_{k^{+}}=\left(k_{+}^{2}-m^{2}+i \epsilon\right)=2 P^{+} x\left[\left(k^{-}+\frac{P^{-}}{2}\right)-\frac{\vec{k}_{T}^{2}+m^{2}}{2 P^{+} x}+\frac{i \epsilon}{2 P^{+} x}\right]
\end{aligned}
$$

We observe that the integration of the function in Eq. (18) with respect to $k^{-}$is different from zero only if $0<x<1$. Indeed, we can perform the Wick rotation in the region

$0<x<1$, where it is well defined according to the positions of the poles determined by Eqs. (25), whereas for $x<0$ and $x>1$ the $\pi$ DA will trivially vanish. For a calculation 


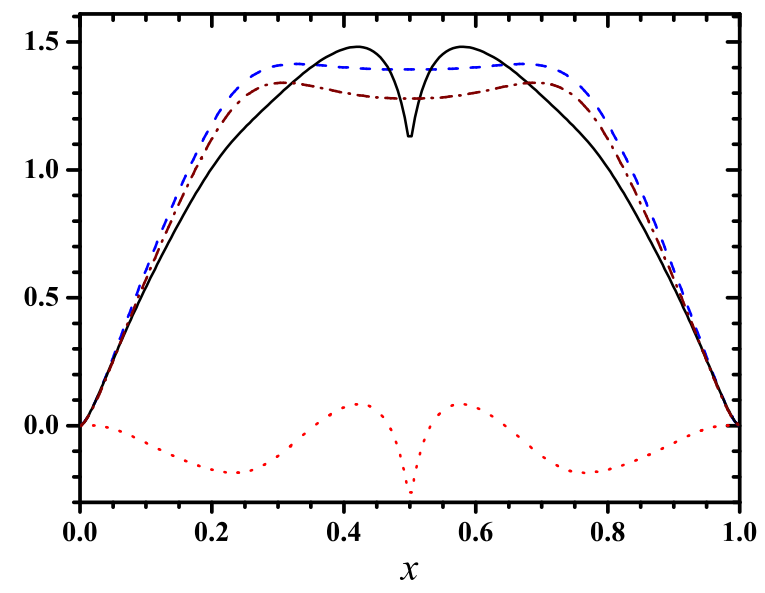

FIG. 2: Pion distribution amplitude. The solid line stands for the $\pi$ DA obtained in the present approach, Eq. (16), while dashed and dotted lines correspond to the contributions given by Eqs. (18) and (19), respectively. The dashed-dotted curve stands for the distribution amplitude defined by Eq. (26), normalized in order to satisfy the sum rule Eq. (5).

performed in Euclidean space (as in our case), the poles lie outside the region of integration, and the loop integrals are formally well defined. However, for $x<0$ or $x>1$ the positions of the poles do not allow us to perform the Wick rotation, thus in these regions the result cannot be connected with the definition of the $\pi$ DA in Minkowski space. Consequently, the integral in Eq. (16) will have physical meaning only for $x \in[0,1]$.

The last, third property becomes the main consistency check for a calculation in Euclidean space. Indeed, the fact that the sum rule is fulfilled when $\phi_{\pi}(x)$ is integrated from 0 to 1 confirms that our $\pi \mathrm{DA}$ has the proper support.

Within the framework of nlNJL models, several authors have used in the definition of the $\pi$ DA the operator $\gamma^{+} \gamma_{5}$ without dressing. In our scheme, this choice would correspond to the following expression for the $\pi \mathrm{DA}$ :

$$
\phi_{\pi}^{(0)}(x)=\frac{2 \sqrt{2} N_{c} g_{\pi q \bar{q}}}{f_{\pi}} \int \frac{d w d^{2} k_{T}}{(2 \pi)^{4}} \frac{g_{k} z_{k_{+}} z_{k_{-}}\left[(1-x) m_{k_{+}}+x m_{k_{-}}\right]}{\left(k_{+}^{2}+m_{k_{+}}^{2}\right)\left(k_{-}^{2}+m_{k_{-}}^{2}\right)} .
$$

It can be seen that in this case the sum rule Eq. (5) is not satisfied. Indeed, in our approach, the usage of Eq. (26) to evaluate the sum rule yields 0.845 instead of 1 . The distribution amplitude given by Eq. (26), properly normalized to satisfy the sum rule [i.e. $\left.\phi_{\pi}^{(0)}(x) / 0.845\right]$, 


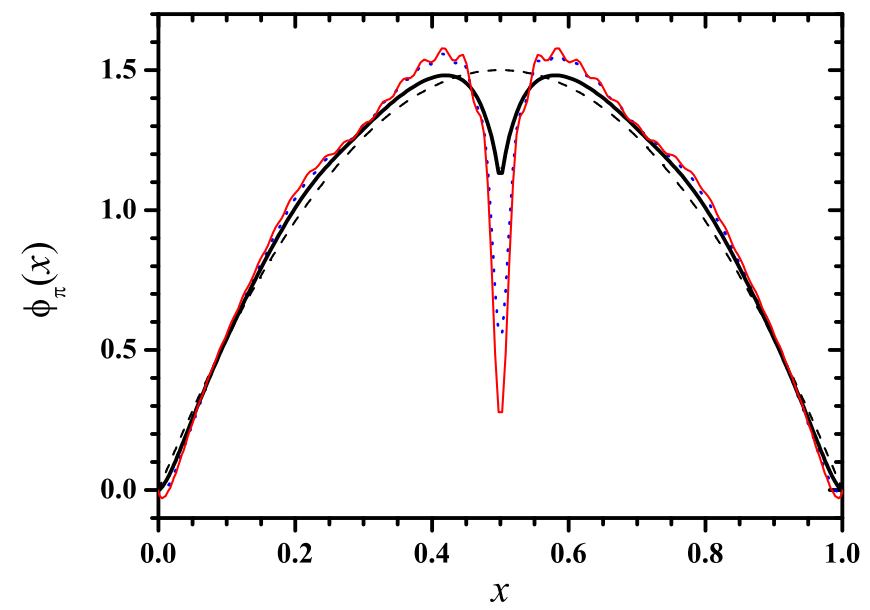

FIG. 3: $\pi$ DA within our model at $\mu=3 \mathrm{GeV}$ (thick solid line), and evolved $\pi \mathrm{DA}$ at $\mu=1 \mathrm{GeV}$, at both LO (dotted line) and NLO (thin solid line). The dashed line corresponds to the asymptotic $\pi$ DA limit.

is also shown in Fig. 2 (dashed-dotted line). We observe that, except for a soft depression in the central part, this result is close to the contribution given by Eq. (18).

Let us consider now the QCD evolution of the $\pi \mathrm{DA}$. A crucial point is the choice of the scale $\mu_{0}$ to be associated to the result provided by the quark model. In our case this value is fixed by that of the lattice calculation used to model the quark propagator. According to Ref. [38], we have to take $\mu_{0}=3 \mathrm{GeV}$, which is a large value compared to the scale $\mu_{0} \sim 1 \mathrm{GeV}$ usually adopted in model calculations.

In Fig. 3 we show the distribution amplitude obtained in our model together with its evolution up to $\mu=1 \mathrm{GeV}$, at LO and NLO. It is seen that the $\pi \mathrm{DA}$ at $\mu_{0}=3 \mathrm{GeV}$ is not far from the asymptotic limit $\phi_{\pi}(x)=6 x(1-x)$. The most significant difference between the results after the $\mathrm{LO}$ and $\mathrm{NLO}$ evolutions of the $\pi \mathrm{DA}$ is that the central minimum decreases significantly; nevertheless, the two maxima do not separate appreciably. As it is expected, the $\pi$ DA moves away from the asymptotic limit. Another important feature of the obtained $\pi \mathrm{DA}$ is that it goes to zero rather fast for $x=0$ and $x=1$, supporting the idea of suppression of the kinematic endpoints [30, 31]. Moreover, this feature is stressed in the evolution towards smaller values of $\mu$, as it should be expected, because the predicted $\pi$ DA lies below the asymptotic one in this region. 


\begin{tabular}{|c|c|c|c|c|c|c|}
\hline $\mathrm{LO}$ & $a_{2}(\mu)$ & $a_{4}(\mu)$ & $a_{6}(\mu)$ & $a_{8}(\mu)$ & $a_{10}(\mu)$ & $a_{12}(\mu)$ \\
\hline$\mu=1 \mathrm{GeV}$ & 0.0047 & -0.0407 & 0.0006 & -0.0185 & 0.0081 & -0.0144 \\
$\mu=2 \mathrm{GeV}$ & 0.0037 & -0.0281 & 0.0004 & -0.0112 & 0.0047 & -0.0080 \\
$\mu=3 \mathrm{GeV}$ & 0.0033 & -0.0238 & 0.0003 & -0.0089 & 0.0036 & -0.0061 \\
\hline
\end{tabular}

TABLE I: Coefficients of the Gegenbauer expansion calculated at LO

\begin{tabular}{|c|c|c|c|c|c|c|}
\hline NLO & $a_{2}(\mu)$ & $a_{4}(\mu)$ & $a_{6}(\mu)$ & $a_{8}(\mu)$ & $a_{10}(\mu)$ & $a_{12}(\mu)$ \\
\hline$\mu=1 \mathrm{GeV}$ & 0.0113 & -0.0482 & -0.0019 & -0.0242 & 0.0081 & -0.0189 \\
$\mu=2 \mathrm{GeV}$ & 0.0048 & -0.0289 & -0.0002 & -0.0117 & 0.0045 & -0.0084 \\
$\mu=3 \mathrm{GeV}$ & 0.0033 & -0.0238 & 0.0003 & -0.0089 & 0.0036 & -0.0061 \\
\hline
\end{tabular}

TABLE II: Coefficients of the Gegenbauer expansion calculated at NLO.

In Table I (II) we quote the first coefficients of the Gegenbauer expansion obtained with our $\pi$ DA at LO (NLO), while in Table III the values obtained by other authors at $\mu=1 \mathrm{GeV}$ are also shown. It is seen that, at variance with the results obtained in other works [19], within our approach the absolute values of the expansion coefficients $a_{n}$ decrease rather slowly with $n$.

\section{LIGHT-CONE WAVE FUNCTION AND $k_{T}$ DEPENDENCE}

The concept of $\pi \mathrm{DA}$ is often associated to that of light-cone wave function (lcwf). If the pion wave function is expanded in terms of Fock states, the first (valence) component,

\begin{tabular}{|l|c|c|c|c|c|c|}
\hline & $a_{2}(\mu)$ & $a_{4}(\mu)$ & $a_{6}(\mu)$ & $a_{8}(\mu)$ & $a_{10}(\mu)$ & $a_{12}(\mu)$ \\
\hline Ref. [34] & 0.10 & 0.10 & 0.10 & 0.034 & 0 & 0 \\
Ref. [19] $(M=350, n=1)$ & 0.114 & 0.015 & 0.001 & 0.0001 & - & - \\
Ref. [19] $(M=350, n=2)$ & 0.066 & -0.027 & -0.017 & -0.006 & - & - \\
Ref. [29] & 0.20 & -0.14 & 0 & 0 & - & - \\
Ref. [36] $(\mu=2 \mathrm{GeV})$ & 0.22 & 0.01 & - & - & - & - \\
\hline
\end{tabular}

TABLE III: Gegenbauer coefficients for the $\pi$ DA given by various authors. The scale is $\mu=1 \mathrm{GeV}$, except in the last row. An exhaustive list of results is given in Refs. [30] and [33]. 
dominant at large $Q^{2}$, is the $\operatorname{lcwf} \Phi_{\pi}^{A}\left(x, k_{T}\right)$, defined by

$$
\begin{aligned}
i \sqrt{2} f_{\pi} \Phi_{\pi}^{A}\left(x, k_{T}\right)= & \int \frac{d z^{-} d^{2} z_{T}}{2 \pi} e^{i P^{+} z^{-}\left(x-\frac{1}{2}\right)-i \vec{k}_{T} \cdot \vec{z}_{T}} \times \\
& \left.\left\langle 0\left|\bar{u}\left(-\frac{z}{2}\right) \gamma^{+} \gamma_{5} d\left(\frac{z}{2}\right)\right| \pi^{-}(P)\right\rangle\right|_{z^{+}=0} .
\end{aligned}
$$

The label $A$, denoting "axial" lcwf, has been used, e.g., in Ref. [53]. When dealing with hard-exclusive processes, the lcwf Eq. (27), integrated with respect to $k_{T}$, can be identified with the $\pi \mathrm{DA}$ [51]. Therefore, in order to carry out a phenomenological analysis, some authors do not distinguish between the $\left(k_{T}\right.$-integrated) lcwf and the $\pi$ DA. In this section we compare the predictions in those works with ours, paying special attention to the results related to the quark transverse momentum $k_{T}$. To this aim, some caveats are in order.

The most direct comparison that could be performed is that between the results obtained in other works for $\Phi_{\pi}^{A}\left(x, k_{T}\right)$, Eq. (27), and those obtained here for the $\pi$ DA, Eq. (16). If we write

$$
\phi_{\pi}(x)=\int \frac{d^{2} k_{T}}{(2 \pi)^{2}} \Phi_{\pi}\left(x, k_{T}\right)
$$

it is natural to identify [c.f. Eq. (16)]

$$
\Phi_{\pi}^{A}\left(x, k_{T}\right) \equiv \Phi_{\pi}\left(x, k_{T}\right)=\frac{2 \sqrt{2} N_{c} g_{\pi q \bar{q}}}{f_{\pi}} \int \frac{d w}{(2 \pi)^{2}} F\left(w, x, k_{T}\right)
$$

On the other hand, in some works the lcwf has been identified with a different quantity, which in our context would correspond to $\Phi_{\pi}^{(0)}\left(x, k_{T}\right)$, obtained from the relation

$$
\phi_{\pi}^{(0)}(x)=\int \frac{d^{2} k_{T}}{(2 \pi)^{2}} \Phi_{\pi}^{(0)}\left(x, k_{T}\right)
$$

with $\phi_{\pi}^{(0)}(x)$ given by Eq. (26). From Eqs. (26) and $(30), \Phi_{\pi}^{(0)}\left(x, k_{T}\right)$ can be cast in the form

$$
\Phi_{\pi}^{(0)}\left(x, k_{T}\right)=N \int d w \frac{g_{k} z_{k_{+}} z_{k_{-}}\left[(1-x) m_{k_{+}}+x m_{k_{-}}\right]}{\left(k_{+}^{2}+m_{k_{+}}^{2}\right)\left(k_{-}^{2}+m_{k_{-}}^{2}\right)},
$$

where $N$ is a normalization factor.

We recall that $\phi_{\pi}^{(0)}(x)$ is the $\pi$ DA evaluated in nlNJL models using the operator $\gamma^{+} \gamma_{5}$ without dressing, while the full $\pi$ DA obtained in the present nlNJL approach includes also other operators carrying different tensor structures, namely $\bar{u}\left(p_{1}+p_{2}\right)^{+}\left(\not p_{1}+\not p_{2}\right) \gamma_{5} d$ and $\bar{u}\left(p_{1}-p_{2}\right)^{+} \gamma_{5} d$, where $p_{1,2}$ are the quark momenta. We emphasize therefore that in the 
present scheme the $\left(k_{T}\right.$-integrated) pion lcwf and the $\pi \mathrm{DA}$ are different quantities. In particular, as it is discussed in the previous section, the latter satisfies exactly the normalization sum rule Eq. (5).

Thus, in the following we will compare $\Phi_{\pi}^{A}\left(x, k_{T}\right)$, evaluated within other approaches, with our results for both the quantities $\Phi_{\pi}\left(x, k_{T}\right)$ and $\Phi_{\pi}^{(0)}\left(x, k_{T}\right)$. It is worth stressing that some predictions concerning the $k_{T}$ dependence could be ultimately related to observables.

Let us consider the quantities

$$
\left\langle k_{T}^{2}\right\rangle_{(0)}=\frac{\int d x d^{2} k_{T} k_{T}^{2}\left|\Phi_{\pi}^{(0)}\left(x, k_{T}\right)\right|^{2}}{\int d x d^{2} k_{T}\left|\Phi_{\pi}^{(0)}\left(x, k_{T}\right)\right|^{2}}
$$

and

$$
\left\langle k_{T}^{2}\right\rangle=\frac{\int d x d^{2} k_{T} k_{T}^{2}\left|\Phi_{\pi}\left(x, k_{T}\right)\right|^{2}}{\int d x d^{2} k_{T}\left|\Phi_{\pi}\left(x, k_{T}\right)\right|^{2}} .
$$

Since $\Phi_{\pi}^{(0)}\left(x, k_{T}\right)$ and $\Phi_{\pi}\left(x, k_{T}\right)$ are probability amplitudes, either $\left\langle k_{T}^{2}\right\rangle$ or $\left\langle k_{T}^{2}\right\rangle_{(0)}$ can be interpreted as the average transverse momentum of the valence quark. For the region of high $Q^{2}$ (i.e., where the lcwf is thought to be the dominant contribution to the pion wave function [51]) this quantity could be accessed in future measurements, performed along the lines proposed in Ref. [52].

In our framework we get $\left\langle k_{T}^{2}\right\rangle_{(0)}^{1 / 2}=270 \mathrm{MeV}$ and $\left\langle k_{T}^{2}\right\rangle^{1 / 2}=260 \mathrm{MeV}$. It is interesting to compare these values with the result obtained in Ref. [36], namely $\left\langle k_{T}^{2}\right\rangle^{1 / 2} \simeq 710 \mathrm{MeV}$, where the average is evaluated considering an axial pion lcwf at a scale $\mu=1 \mathrm{GeV}$. The corresponding $k_{T}$ dependence is given by

$$
\Phi_{\pi}^{A}\left(x, k_{T}\right)=\phi_{\pi}(x) \frac{4 \pi \sigma_{\pi}^{2}}{x(1-x)} \exp \left(-\frac{k_{T}^{2} \sigma_{\pi}^{2}}{x(1-x)}\right)
$$

where $\phi_{\pi}(x)$ is the $\pi$ DA. Although at first sight the results seem to disagree, if we make use of Eqs. (8) and (9) of Ref. [36] in order to determine the value of $\left\langle k_{T}^{2}\right\rangle^{1 / 2}$ at the asymptotic limit, and take for the "traverse size parameter" the value $\sigma_{\pi} \sim 1 \mathrm{GeV}^{-1}$ (upper limit of the range considered in Ref. [36]), we get $\left\langle k_{T}^{2}\right\rangle^{1 / 2} \sim 300 \mathrm{MeV}$. Therefore, our result is found to be somewhat lower but not incompatible with that obtained in Ref. [36].

Now let us also consider a pseudoscalar pion lcwf, $\Phi_{\pi}^{P}\left(x, k_{T}\right)$. The latter has been introduced in Ref. [53], with the aim of obtaining constraints on the lcwf in a light-cone sum rule framework. In order to analyze this function in the context of the nlNJL model, let us 
start by defining the $k_{T}$-integrated pseudoscalar function $\phi_{\pi}^{P}(x)$, which is higher twist with respect to the axial one:

$$
-\frac{2 \sqrt{2}\langle\bar{q} q\rangle}{P^{+} f_{\pi}} \phi_{\pi}^{P}(x)=\left.\int \frac{d z^{-}}{2 \pi} e^{i P^{+} z^{-}\left(x-\frac{1}{2}\right)}\left\langle 0\left|\bar{\psi}\left(-\frac{z}{2}\right) i \gamma_{5} \tau^{-} \psi\left(\frac{z}{2}\right)\right| \pi^{-}(P)\right\rangle\right|_{z^{+}=0, \vec{z}_{\perp}=0} .
$$

It is seen that $\phi_{\pi}^{P}(x)$ fulfills an approximate sum rule, which becomes exact in the chiral limit:

$$
\int_{0}^{1} d x \phi_{\pi}^{P}(x)=1+\mathcal{O}\left(m_{\pi}^{2}\right)
$$

For this function, within the present approach one gets the result

$$
\begin{aligned}
\phi_{\pi}^{P}(x) & =\int \frac{d^{2} k_{T}}{(2 \pi)^{2}} \Phi_{\pi}^{P}\left(x, k_{T}\right) \\
& =-\frac{f_{\pi} \sqrt{2} N_{c}}{\langle\bar{q} q\rangle} g_{\pi q \bar{q}} \int \frac{d w d^{2} k_{T}}{(2 \pi)^{4}} \frac{g_{k} z_{k_{+}} z_{k_{-}}\left(k^{2}+\frac{m_{\pi}^{2}}{4}+m_{k_{+}} m_{k_{-}}\right)}{\left(k_{+}^{2}+m_{k_{+}}^{2}\right)\left(k_{-}^{2}+m_{k_{-}}^{2}\right)}
\end{aligned}
$$

hence the pseudoscalar lcwf will be given by

$$
\Phi_{\pi}^{P}\left(x, k_{T}\right)=-\frac{f_{\pi} \sqrt{2} N_{c}}{\langle\bar{q} q\rangle} g_{\pi q \bar{q}} \int \frac{d w}{(2 \pi)^{2}} \frac{g_{k} z_{k_{+}} z_{k_{-}}\left(k^{2}+\frac{m_{\pi}^{2}}{4}+m_{k_{+}} m_{k_{-}}\right)}{\left(k_{+}^{2}+m_{k_{+}}^{2}\right)\left(k_{-}^{2}+m_{k_{-}}^{2}\right)} .
$$

In addition, with the aim of finding light-cone sum rules, in Refs. [19, 53] the authors also consider the following $k_{T}$ moments of the lcwf $\Phi_{\pi}^{A, P}\left(x, k_{T}\right)$ :

$$
\left\langle k_{T}^{m}\right\rangle_{A, P}=\int d x \frac{d^{2} k_{T}}{(2 \pi)^{2}} k_{T}^{m} \Phi_{\pi}^{A, P}\left(x, k_{T}\right), \quad \text { with } m=2,4 .
$$

It is important to remark that $\Phi_{\pi}^{A, P}\left(x, k_{T}\right)$ are not momentum density distributions. In fact, there is no guarantee that these functions are positive defined. Therefore Eq. (39) is not related to observable quantities and it may be useful only for theoretical considerations. In the present approach, from the analytical expressions in Eqs. (29) and (38) it is seen that for large $k_{T}$ the functions $\Phi_{\pi}^{A, P}\left(x, k_{T}\right)$ behave as

$$
\begin{aligned}
& \Phi_{\pi}^{A}\left(x, k_{T}\right) \underset{k_{T} \rightarrow \infty}{\longrightarrow} k_{T}^{-5}, \\
& \Phi_{\pi}^{P}\left(x, k_{T}\right) \underset{k_{T} \rightarrow \infty}{\longrightarrow} k_{T}^{-3} .
\end{aligned}
$$

In view of these asymptotic behaviors, only the estimate $\left\langle k_{T}^{2}\right\rangle_{A}^{1 / 2}=445 \mathrm{MeV}$ can be obtained, while $\left\langle k_{T}^{4}\right\rangle_{A}$ and $\left\langle k_{T}^{2,4}\right\rangle_{P}$ are not well defined. One has to say that, in our approach, only 
the nonperturbative $k_{T}$ dependence arises naturally from the model calculation. As it is well known, an additional perturbative dependence is found if one takes into account that configurations with two quarks carrying high $k_{T}$ are suppressed due to gluon radiation [2]. This is the origin, for example, of the factor $k_{T}^{2} \sigma_{\pi}^{2}$ in the exponent of Eq. (34). We have considered the possibility of obtaining a prediction for $\left\langle k_{T}^{4}\right\rangle_{A}$ and $\left\langle k_{T}^{2,4}\right\rangle_{P}$ by including a high $k_{T}$ suppression factor such as e.g. these exponential functions in our lcwfs. However, we have found that our results are quite sensitive to the cutoff prescription, hence we are not able to provide a robust prediction for these quantities.

It is worth noticing that the wave functions $\Phi_{\pi}^{P}\left(x, k_{T}\right)$ and $\Phi_{\pi}^{A}\left(x, k_{T}\right)$ are quite different from each other. From Fig. 4, where we have plotted $\phi_{\pi}^{P}(x)$ together with $\phi_{\pi}^{A}(x) \equiv \phi_{\pi}(x)$, we observe that $\phi_{\pi}^{P}(x)$ has less structure than $\phi_{\pi}^{A}(x)$. In fact, $\phi_{\pi}^{P}(x)$ appears to be close to a flat distribution, which corresponds to the asymptotic limit $\phi_{\pi}^{P}(x)=1$. Moreover, the $k_{T}$ dependence is also very different. This can be seen in Fig. 5, where we show our results for

the functions $\Phi_{\pi}^{A}\left(x, k_{T}\right), \Phi_{\pi}^{P}\left(x, k_{T}\right)$ and $\Phi_{\pi}^{(0)}\left(x, k_{T}\right)$ as functions of $k_{T}$ for some definite values of $x$. We also include the results for the axial pion lcwf proposed in Ref. [36], Eq. (34), with $\sigma_{\pi}=0.4 \mathrm{GeV}^{-1}$. In the figure, the results are presented in such a way that the curves corresponding to the lcwf in Eq. (34) have the same value at $k_{T}=0$ for all values of $x$. It is clear that neither the shape nor the size of our functions $\Phi_{\pi}^{A}\left(x, k_{T}\right)$ and $\Phi_{\pi}^{(0)}\left(x, k_{T}\right)$ support the $k_{T}$ dependence proposed in Ref. [36]. Instead, the latter shows a somewhat qualitative agreement with our results for $\Phi_{\pi}^{P}\left(x, k_{T}\right)$, at least, for values of $x$ above say 0.1 .

\section{THE PION-PHOTON TRANSITION FORM FACTOR}

In this section we present the results for the $\pi-\gamma$ TFF obtained within our approach, i.e. via the $\pi \mathrm{DA}$ described in Sec. III. Here we have modified the expression in Eq. (1) by adding sub-leading terms in the expansion in powers of $1 / Q^{2}$. This procedure has been already used in Ref. [17] in order to simulate e.g. contributions coming from higher twist operators. We propose to include two additional terms in the expansion, writing

$$
Q^{2} F\left(Q^{2}\right)=\frac{\sqrt{2} f_{\pi}}{3}\left[\int_{0}^{1} d x T_{H}\left(x, Q^{2}, \mu\right) \phi_{\pi}(x, \mu)+\frac{C}{Q^{2}}+\frac{D}{Q^{4}}\right]
$$

where $C$ and $D$ are constants to be determined by fitting our expression to the experimental data. For the scale $\mu$ we will take $\mu^{2}=Q^{2}$. 


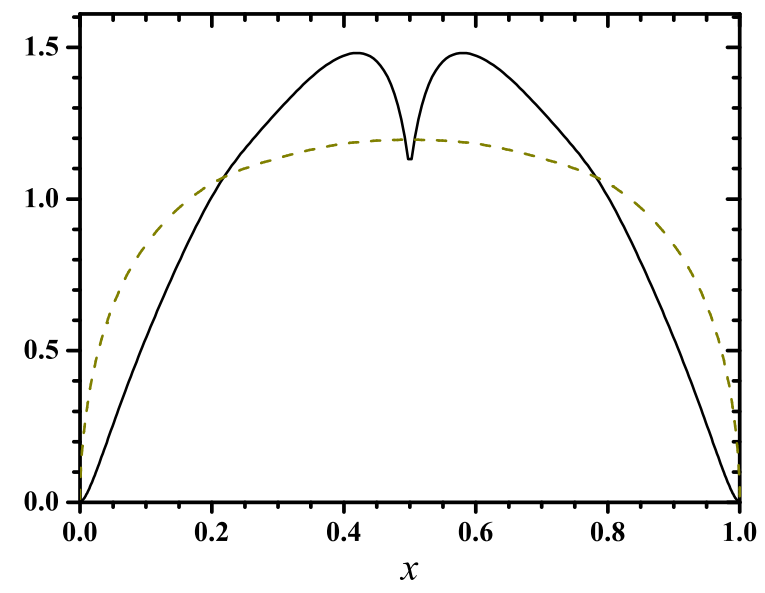

FIG. 4: $\pi$ DA (solid line) and light-cone wave function $\phi_{\pi}^{P}(x)$ (dashed line), Eq. (35), in the nlNJL model.

In Fig. 6 we show our results for $Q^{2} F\left(Q^{2}\right)$. Long-dashed and solid curves correspond to LO and NLO evolutions of the $\pi \mathrm{DA}$, respectively, whereas the dashed-dotted (shortdashed) line stands for the contribution of the term $C / Q^{2}+D / Q^{4}$ at $\mathrm{LO}$ (NLO). The values obtained for the parameters $C$ and $D$ from a fit to all available data (i.e. including the data from CELLO, CLEO, BABAR and BELLE experiments), up to LO and NLO accuracy, are listed in the first row of Table IV. Only the three data with $Q^{2}>1 \mathrm{GeV}^{2}$ of the CELLO Collaboration have been retained in our fit.

Three main conclusions can be outlined from these results: $i$ ) the overall agreement between the fitted curve and the data is not satisfactory; $i i$ ) the values of the parameters $C$ and $D$ are not stable when going from LO to NLO; iii) what is more relevant, the accuracy of the fit is rather worse at NLO than at LO.

As it has been done in Ref. [32], we have also considered separately the inclusion of the data from BABAR and those from BELLE. From Table IV (second row) it is seen that if one excludes the BELLE results from the full data set, the picture does not change appreciably. On the other hand, if one excludes the BABAR data the situation is somehow different (see third row in Table IV): while the agreement with the data gets improved, problems ii) and, in particular, iii), still remain. One can therefore conclude that BELLE data can be easily adjustable in our scheme, especially at LO, and that the corrections arising from NLO 


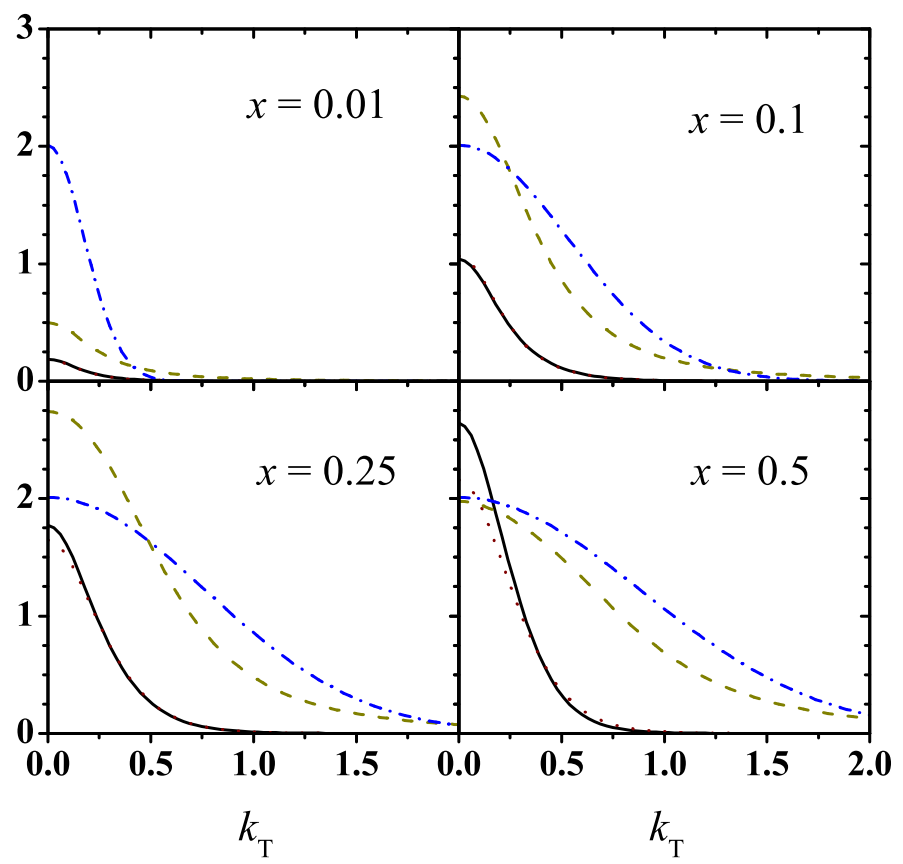

FIG. 5: Solid and dotted lines correspond to the pion axial lcwf $\Phi_{\pi}^{A}\left(x, k_{T}\right)$, Eq. (29), and the function $\Phi_{\pi}^{(0)}\left(x, k_{T}\right)$, Eq. (31), respectively, both multiplied by a factor $x(1-x) /\left[10 \phi_{\pi}(x)\right]$. The dashed line stands for the pion pseudoscalar lcwf $\Phi_{\pi}^{P}\left(x, k_{T}\right)$, Eq. (38), while the dashed-dotted line corresponds to the pion axial lcwf considered in Ref. [36], Eq. (34), both multiplied by $x(1-$ $x) / \phi_{\pi}(x)$. We remark that the results for the first two quantities are rescaled by a factor of 10 with respect to the last two.

contributions to the evolution equations go in the wrong direction, in all cases under study.

In order to test this last statement, we have checked what happens if, instead of the $\pi \mathrm{DA}$ from our nlNJL model, we take as input a flat distribution $\phi_{\pi}(x)=1$. In order to avoid singularities, in this case we modify the kernel $T_{H}\left(x, Q^{2}, \mu\right)$ introducing a new parameter $M[8]:$

$$
T_{H}^{\mathrm{NLO}}\left(x, Q^{2}, \mu\right)=\frac{1}{x+\frac{M^{2}}{Q^{2}}}\left\{1+C_{F} \frac{\alpha_{\mathrm{s}}(\mu)}{4 \pi}\left[\ln ^{2} x-\frac{x \ln x}{1-x}-9+(3+2 \ln x) \ln \frac{Q^{2}}{\mu^{2}}\right]\right\} .
$$




\begin{tabular}{|c|c|c|c|c|c|c|c|}
\hline$\pi$ DA & Data set & Accuracy & $C\left[\mathrm{GeV}^{2}\right]$ & $D\left[\mathrm{GeV}^{4}\right]$ & $\mathrm{M}[\mathrm{GeV}]$ & $\mathrm{n}^{\circ}$ points & $\chi^{2} / \mathrm{n}^{\circ}$ points \\
\hline \multirow{2}{*}{ nlNJL } & Cello+Cleo+Belle+Babar & LO & -1.82 & 0.29 & - & 50 & 1.9 \\
& & NLO & 1.56 & -3.09 & - & 50 & 3.5 \\
\hline \multirow{2}{*}{ nlNJL } & Cello+Cleo+Babar & LO & -1.80 & 0.26 & - & 35 & 2.4 \\
& & NLO & 1.49 & -2.95 & - & 35 & 4.3 \\
\hline \multirow{2}{*}{ nlNJL } & Cello+Cleo+Belle & LO & -2.01 & 0.65 & - & 33 & 0.61 \\
& & NLO & 0.90 & -1.91 & - & 33 & 1.09 \\
\hline \multirow{2}{*}{ flat } & Cello+Cleo+Belle+Babar & LO & 1.82 & -1.50 & 0.76 & 50 & 0.91 \\
& & NLO & 1.47 & -1.08 & 0.57 & 50 & 0.96 \\
\hline
\end{tabular}

TABLE IV: Values of the parameters $C, D$ and $M$ [see Eqs. (40) and (41)], obtained from the fits to different experimental data sets for the $\pi \mathrm{TFF}$. The first three rows correspond to the $\pi \mathrm{DA}$ calculated within the nINJL model, while entries in the last row are obtained from a flat $\pi$ DA.

We take here the scale $\mu_{0}=1 \mathrm{GeV}$ [17], at which one assumes that the quark model provides a good description of low energy physics.

In Fig. 7 (see also fourth row of Table IV) we show the results for $Q^{2} F\left(Q^{2}\right)$ obtained after inserting the function in Eq. (41) into Eq. (40), for a flat distribution $\phi_{\pi}(x)=1$ [at the LO, only the first term into the brackets in Eq. (41) has to be considered]. It is seen that the agreement with the full set of experimental data becomes improved with respect to the previous analyses (one should notice anyway that a further parameter, $M$, has been included), and that the parameters of the fit are more stable when passing from LO to NLO. We stress, however, that the important conclusion iii) stated in the previous cases still holds: the inclusion of NLO corrections does not help to describe the experimental data. This becomes more evident for virtualities $Q^{2}$ above $10 \mathrm{GeV}^{2}$.

Due to the regularization of $T_{H}$ in the limit $x \rightarrow 0$, it is difficult to compare our results for the flat distribution with those obtained within approaches based on the parametrization of the Gegenbauer expansion. For instance, one could define an effective $\pi \mathrm{DA} \phi_{\pi}^{\text {eff }}(x, Q)$ by the relation

$$
\left(\frac{1}{x}+\frac{1}{1-x}\right) \phi_{\pi}^{\mathrm{eff}}(x, Q)=\left(\frac{1}{x+\frac{M^{2}}{Q^{2}}}+\frac{1}{1-x+\frac{M^{2}}{Q^{2}}}\right) \phi_{\pi}(x, Q)
$$

now the problem is that it would not be guaranteed that $a_{0}(Q)=1$ in the Gegenbauer 


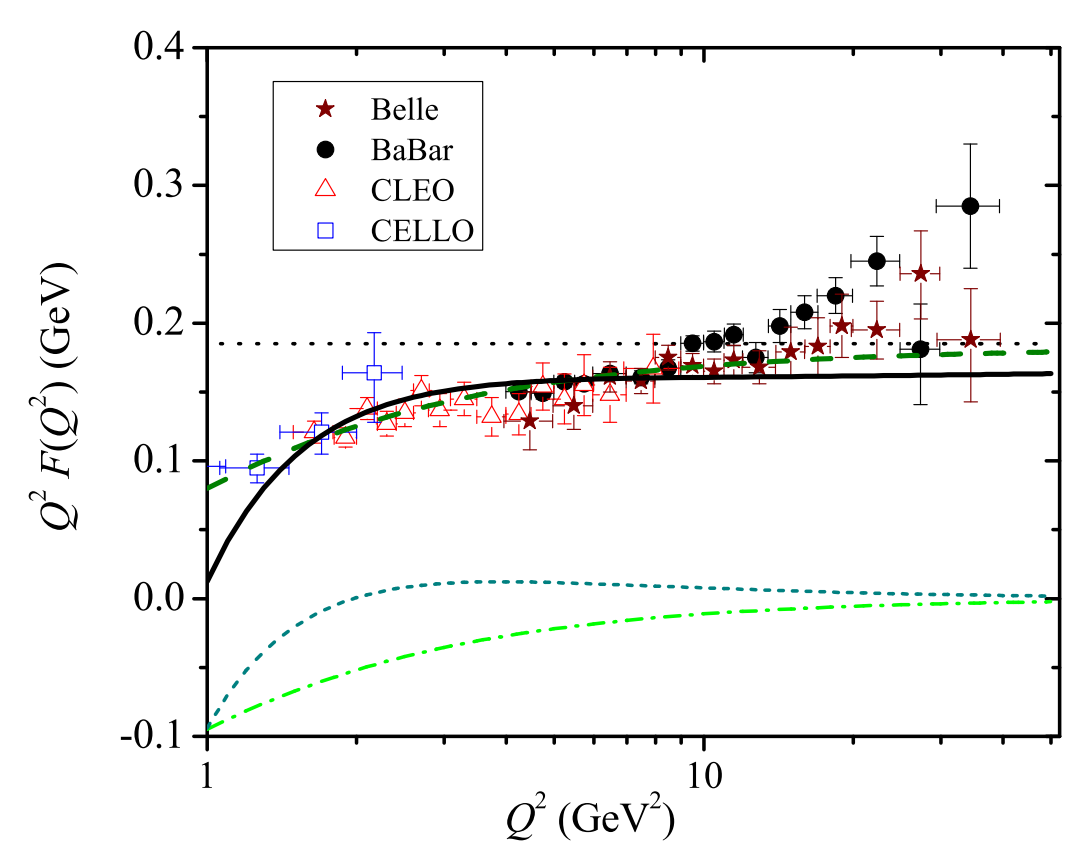

FIG. 6: Values of $Q^{2} F\left(Q^{2}\right)$, Eq. (40), at LO (long-dashed line) and NLO (solid line), in comparison with experimental data. Dashed-dotted and short-dashed curves show the contributions given by the term $C / Q^{2}+D / Q^{4}$ at $\mathrm{LO}$ and NLO, respectively, while the horizontal dotted line indicates the asymptotic QCD limit.

expansion, Eq. (21). Actually, if we assume a flat distribution $\phi_{\pi}\left(x, \mu_{0}\right)=1$, we get

$$
\int_{0}^{1} d x \phi_{\pi}^{\mathrm{eff}}\left(x, \mu_{0}\right)=1+2 \frac{M^{2}}{\mu_{0}^{2}}-2 \frac{M^{2}}{\mu_{0}^{2}}\left(1+\frac{M^{2}}{\mu_{0}^{2}}\right) \log \left(1+\frac{\mu_{0}^{2}}{M^{2}}\right),
$$

which is equal to 0.33 for $M=0.76 \mathrm{GeV}$ and to 0.44 for $M=0.58 \mathrm{GeV}$. Therefore, we can not compare the coefficients in the Gegenbauer expansion with those obtained in Tables I, II and III.

Finally, it is interesting to notice that the conclusion concerning the NLO corrections is also valid for the asymptotic behavior of the $\pi$ DA. Indeed, taking $\phi_{\pi}(x)=6 x(1-$ $x)$ we find, at the $\mathrm{LO}, Q^{2} F\left(Q^{2}\right)=\sqrt{2} f_{\pi}=0.185 \mathrm{GeV}$, and at the NLO, $Q^{2} F\left(Q^{2}\right)=$ $\sqrt{2} f_{\pi}\left[1-0.53 \alpha_{\mathrm{s}}\left(Q^{2}\right)\right]=0.161(0.164) \mathrm{GeV}$ for $Q^{2}=10(20) \mathrm{GeV}^{2}$. Therefore, the NLO correction reduces the $\pi \mathrm{TFF}$ by about a $13 \%$, in a direction which is opposite to that of the data. 


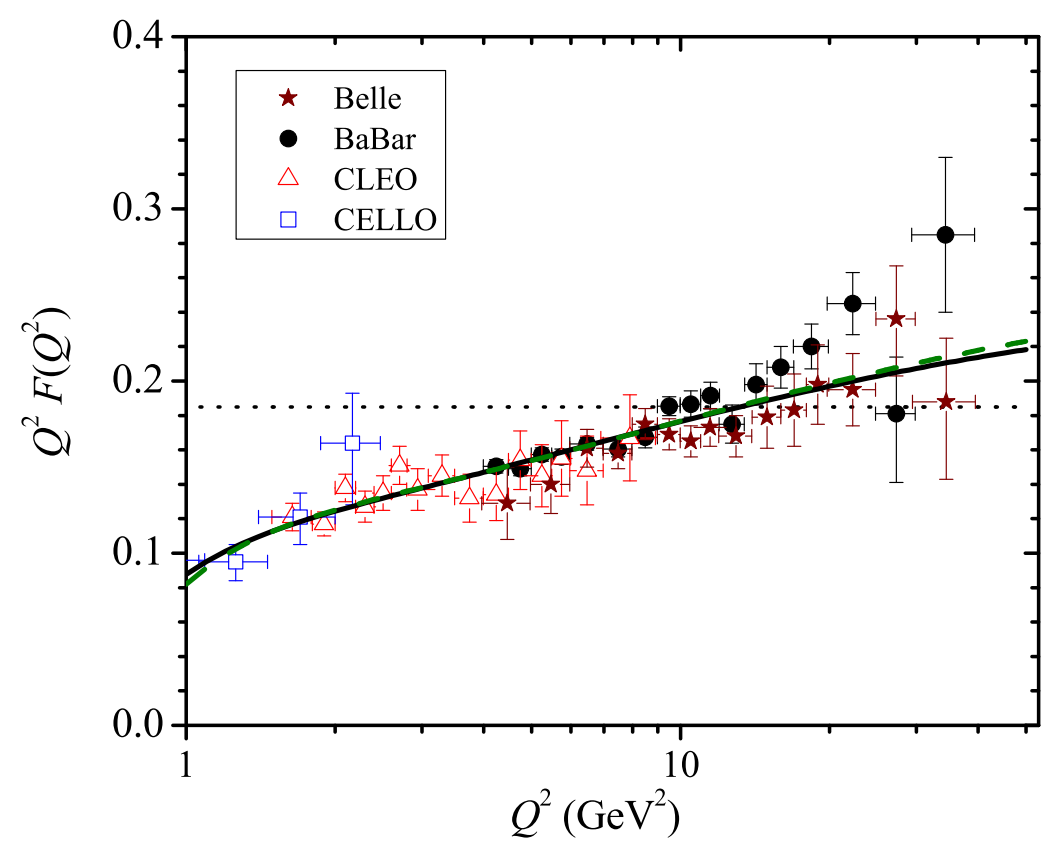

FIG. 7: Values of $Q^{2} F\left(Q^{2}\right)$ obtained from a flat pion distribution amplitude, at LO (dashed line) and NLO (dashed line), in comparison with experimental data. The horizontal dotted line indicates the asymptotic QCD limit.

\section{CONCLUSIONS}

In this work, the $\pi \mathrm{DA}$ and the associated $\pi \mathrm{TFF}$ have been evaluated within the framework of a nonlocal Nambu-Jona-Lasinio model that has been shown to successfully describe several pion observables $[47,48]$. In this approach, the couplings between nonlocal quark currents ensure the preservation of chiral, Poincaré and local electromagnetic gauge invariances. The three main ingredients of the calculation are the description of the pion as a bound state of a Bethe-Salpeter equation, the usage of a prescription for the introduction of the electroweak interaction vertices and, eventually, the quark propagator, which shows the momentum dependence obtained in lattice QCD. The calculated $\pi$ DA has to be therefore associated to the momentum scale of the lattice data, namely $3 \mathrm{GeV}$ [38]. In general, the comparison of any observable related to the $\pi \mathrm{DA}$ (as e.g. the $\pi \mathrm{TFF}$ ) with experimental data will require a perturbative evolution of the results obtained at this reference scale. Here this 
evolution has been carried out up to NLO accuracy.

Since the theoretical framework respects all basic symmetries, our $\pi \mathrm{DA}$ is shown to fulfill three fundamental properties: it has the correct symmetry in the quark momentum fraction, it is defined in the proper support and, above all, it turns out to be naturally normalized, a feature which is imposed in other schemes [19, 22, 29-36]. It is seen that our $\pi \mathrm{DA}$, already at the scale of $3 \mathrm{GeV}$, is not far from the asymptotic distribution $\phi_{\pi}(x)=$ $6 x(1-x)$. In fact, we find that the genuine nonlocal contributions push the result towards this asymptotic behavior. Moreover, the pseudoscalar pion distribution amplitude is also found to approach its corresponding asymptotic limit $\phi_{\pi}^{P}(x)=1$. Another outcome of our results is that when the $\pi \mathrm{DA}$ is expanded in Gegenbauer polynomials, in contrast with other calculations $[19,33,34]$ we find that the absolute values of the corresponding coefficients $a_{n}$ decrease rather slowly with $n$.

The last part of the paper is devoted to phenomenological considerations. Our results for the functions $\Phi_{\pi}\left(x, k_{T}\right)$, where $k_{T}$ is the quark transverse momentum, are compared to those obtained within a light-cone wave function approach. It is found that the $k_{T}$ dependence obtained in our framework turns out to be rather different from that calculated in other works [36]. This feature could in principle be checked in future experiments. Concerning the evaluation of the $\pi \mathrm{TFF}$, we have found that NLO corrections in general lead to a suppression of $Q^{2} F\left(Q^{2}\right)$, which represents a problem towards the explanation of the already challenging experimental scenario. In particular, in our nlNJL approach (which is based on the evaluation of standard diagrams, and considers just general assumptions such as chiral symmetry and lattice results), it is very problematic to obtain a $\pi \mathrm{TFF}$ that crosses the asymptotic limit as suggested by the pattern of the BABAR data. This feature of our results is common to several other calculations (see e.g. Refs. $[23,25,26,32]$ ). It should be stressed that the present nlNJL scheme is quite severely constrained and, thus, it is not easy to address a strategy to reconcile it with the present status of the $\pi \mathrm{TFF}$ measurements. A basic theoretical input of our calculation is represented by the lattice data used to parameterize the quark propagator $[38,39]$. In this sense, it appears that a better description of the presently available $\pi \mathrm{TFF}$ data within the present scheme would require that lattice results were significantly updated in forthcoming analyses. 


\section{Acknowledgements}

We thank A. Pimikov for a critical reading of the manuscript. This work has been partially funded by the Spanish MCyT (and EU FEDER) under contract FPA2010-21750-C02-01 and AIC10-D-000588, by Consolider Ingenio 2010 CPAN (CSD2007-00042), by Generalitat Valenciana: Prometeo/2009/129, by the European Integrated Infrastructure Initiative HadronPhysics3 (Grant number 283286), by CONICET (Argentina) under grants \# PIP 00682 and PIP 02495, and by ANPCyT (Argentina) under grant \# PICT-2011-0113.

\section{Appendix A: Derivation of the $\pi$ DA in the nonlocal NJL model}

In this Appendix we provide some details on the obtention of the $\pi \mathrm{DA}$ in Eq. (16). We start with the Euclidean action in Eq. (6), and include a coupling with an external axial gauge field $a_{\mu}$, as described in Sect. IIB. In order to deal with meson degrees of freedom, it is convenient to bosonize the fermionic theory by introducing scalar and pseudoscalar fields $\sigma_{1,2}(y)$ and $\vec{\pi}(y)$ and integrating out the fermion fields. This bosonized action can be written as $[47,48]$

$$
S^{\text {bos }}=-\ln \operatorname{det} \mathcal{D}+\frac{1}{2 G_{S}} \int d^{4} y\left[\sigma_{1}(y) \sigma_{1}(y)+\sigma_{2}(y) \sigma_{2}(y)+\vec{\pi}(y) \cdot \vec{\pi}(y)\right],
$$

where

$$
\begin{aligned}
\mathcal{D}\left(y+\frac{z}{2}, y-\frac{z}{2}\right)= & \gamma_{0} W\left(y+\frac{z}{2}, y\right) \gamma_{0}\left\{\delta^{(4)}(z)\left[-i \not \partial+m_{c}\right]+\right. \\
& {\left.\left[\mathcal{G}(z)\left[\sigma_{1}(y)+i \vec{\tau} \cdot \vec{\pi}(y)\right]+\mathcal{F}(z) \sigma_{2}(y) \frac{i \overleftrightarrow{\phi}}{2 \varkappa_{p}}\right]\right\} W\left(y, y-\frac{z}{2}\right)(}
\end{aligned}
$$

As usual we assume that the fields $\sigma_{1,2}$ have nontrivial translational invariant mean field values $\bar{\sigma}_{1}$ and $\varkappa_{p} \bar{\sigma}_{2}$, while the mean field values of pseudoscalar fields $\pi_{i}$ are zero. Thus we write

$$
\sigma_{1}(y)=\bar{\sigma}_{1}+\delta \sigma_{1}(y), \quad \sigma_{2}(y)=\varkappa_{p} \bar{\sigma}_{2}+\delta \sigma_{2}(y), \quad \vec{\pi}(y)=\delta \vec{\pi}(y)
$$

Replacing in the bosonized effective action and expanding in powers of meson fluctuations and the external field $a_{\mu}$ we obtain

$$
S^{\text {bos }}=S_{\mathrm{MFA}}+S_{\mathrm{quad}}+S_{\pi a}+\ldots
$$


where only the terms relevant for our calculation have been explicitly written. Here the mean field action per unit volume reads

$$
S_{\mathrm{MFA}}=\frac{1}{2 G_{S}}\left(\bar{\sigma}_{1}^{2}+\varkappa_{p}^{2} \bar{\sigma}_{2}^{2}\right)-4 N_{c} \int \frac{d^{4} p}{(2 \pi)^{4}} \ln \mathcal{D}_{0}
$$

with $\mathcal{D}_{0}=\left(-\not p+m_{p}\right) / z_{p}$, see Eqs. (8) and (9) in Sect. IIB.

The minimization of $S_{\mathrm{MFA}}$ with respect to $\bar{\sigma}_{1,2}$ leads to the corresponding DysonSchwinger equations, which together with Eqs. (9) and (11) allow to determine the values of $G_{S}$ and $\varkappa_{p}$. The quadratic piece of the bosonic Euclidean action can be written as

$$
S_{E}^{\text {quad }}=\frac{1}{2} \int \frac{d^{4} p}{(2 \pi)^{4}} \sum_{M=\sigma, \sigma^{\prime}, \pi} G_{M}\left(p^{2}\right) \delta M(p) \delta M(-p),
$$

where the fields $\sigma$ and $\sigma^{\prime}$ are scalar meson mass eigenstates, defined in such a way that there is no $\sigma-\sigma^{\prime}$ mixing at the level of the quadratic action. The explicit expressions for the oneloop integrals $G_{M}\left(p^{2}\right)$, as well as those of the above mentioned Dyson-Schwinger equations, can be found in Ref. [47]. Meson masses can be obtained by solving the associated BetheSalpeter equations $G_{M}\left(-m_{M}^{2}\right)=0$, while on-shell meson-quark coupling constants $g_{M q \bar{q}}$ are given by

$$
g_{M q \bar{q}}{ }^{-2}=\left.\frac{d G_{M}\left(p^{2}\right)}{d p^{2}}\right|_{p^{2}=-m_{M}^{2}} .
$$

Finally, the bilinear piece in $\delta \pi$ and $a_{\mu}$ fields $S_{\pi a}$ in Eq. (A4) reads

$$
S_{\pi a}=\operatorname{Tr}\left[\begin{array}{llll}
\mathcal{D}_{0}^{-1} & D_{\pi} & \mathcal{D}_{0}^{-1} & D_{a}
\end{array}\right]+\operatorname{Tr}\left[\mathcal{D}_{0}^{-1} D_{\pi a}\right]
$$

where, $\mathcal{D}_{\pi}, \mathcal{D}_{a}$ and $\mathcal{D}_{\pi a}$ stand for the terms in the expansion of Eq. (A2) that are linear in $\delta \pi_{i}$ and/or $a_{\mu}$. The corresponding expressions are long and will not be quoted here. The $\pi$ DA within the nINJL model can then be obtained by taking the functional derivative of $S_{\pi a}$ with respect to $\delta \pi_{i}$ and $a_{\mu}$. It is important to note that due to the bilocal character of the gauge field $a_{\mu}$ associated with the current in Eq. (3) an extra delta function appears in momentum space. Namely, while for the local case we would have

$$
\int d^{4} x \bar{\psi}(y) \Gamma \psi(y) e^{i q \cdot y}=\int \frac{d^{4} p_{1}}{(2 \pi)^{4}} \frac{d^{4} p_{2}}{(2 \pi)^{4}}(2 \pi)^{4} \delta^{(4)}\left(p_{2}+q-p_{1}\right) \bar{\psi}_{p_{2}} \Gamma \psi_{p_{1}}
$$

for a bilocal current of the type appearing in Eq.(3) we have

$$
\begin{aligned}
\left.\int \frac{d \xi^{-}}{2 \pi} \int d^{4} x \bar{\psi}(y-\xi / 2) \Gamma \psi(y+\xi / 2)\right|_{\xi^{+}=0, \vec{\xi}_{T}=0} e^{i q \cdot y} e^{i P^{+} \xi^{-} x} \\
\quad=\int \frac{d^{4} p_{1}}{(2 \pi)^{4}} \frac{d^{4} p_{2}}{(2 \pi)^{4}}(2 \pi)^{4} \delta^{(4)}\left(p_{2}+q-p_{1}\right) \delta\left(P^{+} x-\frac{p_{1}^{+}+p_{2}^{+}}{2}\right) \bar{\psi}_{p_{2}} \Gamma \psi_{p_{1}}
\end{aligned}
$$




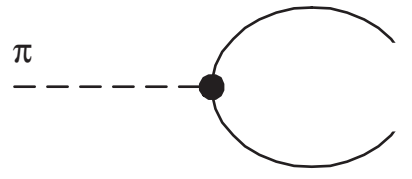

(a)

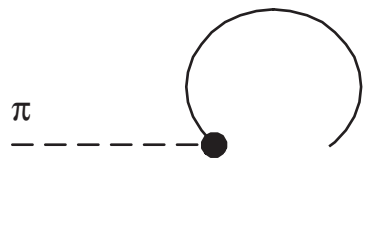

(b)

FIG. 8: Diagrammatic representation of the contributions to the $\pi$ DA. As usually, in diagram (a) the struck quark connects the two open quark lines, whereas in diagram (b) the struck quark goes from the open quark line to the pion-quark vertex.

where $\Gamma$ represents an operator carrying Dirac and flavor indices. In this way, besides the delta function related to four-momentum conservation one has an extra one-dimensional delta that involves the + components of the momenta. The latter can be worked out in Minkowski space [e.g. by integrating over the $z$ component of the momentum $k^{\mu} \equiv$ $\left.\frac{1}{2}\left(p_{1}+p_{2}\right)^{\mu}\right]$, going then back to Euclidean space.

The contributions coming from the two terms in Eq. (A8) can be represented diagrammatically as shown in Fig. 8, where Diag. (a) corresponds to the first term and Diag. (b) to the second one. Regarding the expressions in Eqs. (17-19), Diag. (b) gives rise to the last term of $F_{2}$ [see Eq. (19)] while Diag. (a) accounts for $F_{1}$ and the remaining terms in $F_{2}$.

\section{Appendix B: Renormalization factors for the QCD evolution of the $\pi$ DA}

We quote here the expressions for the renormalization factors $E_{n}^{\mathrm{LO}}, E_{n}^{\mathrm{NLO}}$ and $d_{n}^{k}$ needed to calculate the evolution of the coefficients $a_{n}(\mu)$ in Eqs. (23) and (24). One has

$$
\begin{aligned}
E_{n}^{\mathrm{LO}}\left(\mu, \mu_{0}\right) & =\left[\frac{\alpha_{\mathrm{s}}(\mu)}{\alpha_{\mathrm{s}}\left(\mu_{0}\right)}\right]^{\gamma_{n}^{(0)} /\left(2 \beta_{0}\right)}, \\
E_{n}^{\mathrm{NLO}}\left(\mu, \mu_{0}\right) & =E_{n}^{\mathrm{LO}}\left(\mu, \mu_{0}\right)\left[1+\frac{\alpha_{\mathrm{s}}(\mu)-\alpha_{\mathrm{s}}\left(\mu_{0}\right)}{8 \pi} \frac{\gamma_{n}^{(0)}}{\beta_{0}}\left(\frac{\gamma_{n}^{(1)}}{\gamma_{n}^{(0)}}-\frac{\beta_{1}}{\beta_{0}}\right)\right],
\end{aligned}
$$

where $\beta_{0}\left(\beta_{1}\right)$ and $\gamma_{n}^{(0)}\left(\gamma_{n}^{(1)}\right)$ are the LO (NLO) coefficients of the QCD $\beta$-function and the anomalous dimensions, respectively. The first two coefficients of the $\beta$-function are

$$
\beta_{0}=11-\frac{2}{3} n_{f}, \quad \beta_{1}=102-\frac{38}{3} n_{f},
$$


where $n_{f}$ is the number of flavors (we take here $n_{f}=4$ ). For the evolution of the strong coupling constant $\alpha_{s}$ we use

$$
\alpha_{s}(\mu)=\frac{4 \pi}{\beta_{0} \ln \left(\mu^{2} / \Lambda^{2}\right)}\left\{1-\frac{\beta_{1}}{\beta_{0}^{2}} \frac{\ln \left[\ln \left(\mu^{2} / \Lambda^{2}\right)\right]}{\ln \left(\mu^{2} / \Lambda^{2}\right)}\right\},
$$

taking $\Lambda=0.224 \mathrm{GeV}(\Lambda=0.326 \mathrm{GeV})$ if the calculation is carried out at the LO (NLO). The anomalous dimensions $\gamma_{n}^{(0)}$ are given by

$$
\gamma_{n}^{(0)}=2 C_{F}\left(1-\frac{2}{(n+1)(n+2)}+4 \sum_{m=2}^{n+1} \frac{1}{m}\right)
$$

while analytical expressions for $\gamma_{n}^{(1)}$ can be found in Refs. [54, 55].

On the other hand, the off-diagonal mixing coefficients $d_{n}^{k}$ in Eq. (24) are given by:

$$
d_{n}^{k}\left(\mu, \mu_{0}\right)=\frac{M_{n}^{k}}{\gamma_{n}^{(0)}-\gamma_{k}^{(0)}-2 \beta_{0}}\left\{1-\left[\frac{\alpha_{\mathrm{s}}(\mu)}{\alpha_{\mathrm{s}}\left(\mu_{0}\right)}\right]^{\left[\gamma_{n}^{(0)}-\gamma_{k}^{(0)}-2 \beta_{0}\right] / 2 \beta_{0}}\right\} .
$$

Here the matrix $M_{n}^{k}$ is defined as

$$
\begin{aligned}
M_{n}^{k} & =\frac{(k+1)(k+2)(2 n+3)}{(n+1)(n+2)}\left[\gamma_{n}^{(0)}-\gamma_{k}^{(0)}\right] \\
& \times\left\{\frac{8 C_{F} A_{n}^{k}-\gamma_{k}^{(0)}-2 \beta_{0}}{(n-k)(n+k+3)}+4 C_{F} \frac{\left.A_{n}^{k}-S_{1}(n+1)\right)}{(k+1)(k+2)}\right\},
\end{aligned}
$$

where

$$
A_{n}^{k}=S_{1}\left(\frac{n+k+2}{2}\right)-S_{1}\left(\frac{n-k-2}{2}\right)+2 S_{1}(n-k-1)-S_{1}(n+1),
$$

with

$$
S_{1}(n)=\sum_{j=1}^{n} \frac{1}{j} .
$$

Numerical values of the coefficients $M_{n}^{k}$ for $n \leq 12$ are given in Ref. [33].

[1] A. V. Efremov and A. V. Radyushkin, Phys. Lett. B 94, 245 (1980).

[2] G. P. Lepage and S. J. Brodsky, Phys. Lett. B 87, 359 (1979); Phys. Rev. D 22, 2157 (1980).

[3] H. J. Behrend et al. [CELLO Collaboration], Z. Phys. C 49, 401 (1991).

[4] J. Gronberg et al. [CLEO Collaboration], Phys. Rev. D 57, 33 (1998) [arXiv:hep-ex/9707031]. 
[5] B. Aubert et al. [BABAR Collaboration], Phys. Rev. D 80, 052002 (2009) [arXiv:0905.4778 [hep-ex]].

[6] S. Uehara et al. [Belle Collaboration], Phys. Rev. D 86, 092007 (2012) [arXiv:1205.3249 [hep$\mathrm{ex}]$.

[7] P. del Amo Sanchez et al. [BABAR Collaboration], Phys. Rev. D84, 052001 (2011) [arXiv:1101.1142 [hep-ex]].

[8] A. V. Radyushkin, Phys. Rev. D 80, 094009 (2009) [arXiv:0906.0323 [hep-ph]].

[9] M. V. Polyakov, JETP Lett. 90, 228 (2009) [arXiv:0906.0538 [hep-ph]].

[10] V. L. Chernyak and A. R. Zhitnitsky, Nucl. Phys. B 201, 492 (1982) [Erratum-ibid. B 214, 547 (1983)]; Phys. Rept. 112, 173 (1984).

[11] L. Del Debbio, Few Body Syst. 36, 77 (2005).

[12] V. M. Braun et al., Phys. Rev. D 74, 074501 (2006) [arXiv:hep-lat/0606012].

[13] E. Ruiz Arriola and W. Broniowski, Phys. Rev. D 66, 094016 (2002) [arXiv:hep-ph/0207266].

[14] A. Courtoy and S. Noguera, Phys. Rev. D 76, 094026 (2007) [arXiv:0707.3366 [hep-ph]].

[15] A. Courtoy, Ph. D. Thesis, Valencia University, 2009 [arXiv:1010.2974 [hep-ph]].

[16] E. Ruiz Arriola and W. Broniowski, Phys. Rev. D 67, 074021 (2003) [arXiv:hep-ph/0301202].

[17] S. Noguera and V. Vento, Eur. Phys. J. A 46, 197 (2010) [arXiv:1001.3075 [hep-ph]]; Eur. Phys. J. A 48, 143 (2012) [arXiv:1205.4598 [hep-ph]].

[18] S. Noguera and S. Scopetta, Phys. Rev. D 85, 054004 (2012) [arXiv:1110.6402 [hep-ph]].

[19] M. Praszalowicz and A. Rostworowski, Phys. Rev. D 64, 074003 (2001); M. Praszalowicz and A. Rostworowski, Phys. Rev. D 66, 054002 (2002) [arXiv:hep-ph/0111196].

[20] S. -i. Nam and H. -Ch. Kim, Phys. Rev. D 74, 076005 (2006) [hep-ph/0609267]; S. -i. Nam, H. -Ch. Kim, A. Hosaka and M. M. Musakhanov, Phys. Rev. D 74, 014019 (2006) [hep$\mathrm{ph} / 0605259]$.

[21] A. E. Dorokhov, JETP Lett. 92, 707 (2010).

[22] A. E. Dorokhov and E. A. Kuraev, Phys. Rev. D 88, 014038 (2013) [arXiv:1305.0888 [hep-ph]].

[23] B. El-Bennich, J. P. B. C. de Melo and T. Frederico, Few Body Syst. 54, 1851 (2013) [arXiv:1211.2829 [nucl-th]].

[24] S. J. Brodsky and G. F. de Teramond, Phys. Rev. D 78, 025032 (2008) [arXiv:0804.0452 [hep-ph]].

[25] S. J. Brodsky, F. -G. Cao and G. F. de Teramond, Phys. Rev. D 84, 075012 (2011) 
[arXiv:1105.3999 [hep-ph]]; S. J. Brodsky, F. -G. Cao and G. F. de Teramond, Phys. Rev. D 84, 033001 (2011) [arXiv:1104.3364 [hep-ph]].

[26] H. L. L. Roberts, C. D. Roberts, A. Bashir, L. X. Gutierrez-Guerrero and P. C. Tandy, Phys. Rev. C 82, 065202 (2010) [arXiv:1009.0067 [nucl-th]].

[27] L. Chang, I. C. Cloet, J. J. Cobos-Martinez, C. D. Roberts, S. M. Schmidt and P. C. Tandy, Phys. Rev. Lett. 110, 132001 (2013) [arXiv:1301.0324 [nucl-th]].

[28] I. C. Clot, L. Chang, C. D. Roberts, S. M. Schmidt and P. C. Tandy, Phys. Rev. Lett. 111, 092001 (2013) [arXiv:1306.2645 [nucl-th]]; J. Segovia, L. Chang, I. C. Cloet, C. D. Roberts, S. M. Schmidt and H. -s. Zong, arXiv:1311.1390 [nucl-th].

[29] A. P. Bakulev, S. V. Mikhailov and N. G. Stefanis, Phys. Lett. B 508, 279 (2001) [Erratumibid. B 590, 309 (2004)] [hep-ph/0103119].

[30] S. V. Mikhailov and N. G. Stefanis, Nucl. Phys. B 821, 291 (2009) [arXiv:0905.4004 [hep-ph]].

[31] S. V. Mikhailov, A. V. Pimikov and N. G. Stefanis, Phys. Rev. D 82, 054020 (2010) [arXiv:1006.2936 [hep-ph]].

[32] A. P. Bakulev, S. V. Mikhailov, A. V. Pimikov and N. G. Stefanis, Phys. Rev. D 84, 034014 (2011) [arXiv:1105.2753 [hep-ph]]; Phys. Rev. D 86, 031501 (2012) [arXiv:1205.3770 [hep-ph]]; Phys. Rev. D 87, 094025 (2013) [arXiv:1202.1781 [hep-ph]]; Nucl. Phys. Proc. Suppl. 225-227, $146(2012)$.

[33] S. S. Agaev, V. M. Braun, N. Offen and F. A Porkert, Phys. Rev. D 83, 054020 (2011) [arXiv:1012.4671 [hep-ph]].

[34] S. S. Agaev, V. M. Braun, N. Offen and F. A Porkert, Phys. Rev. D 86, 077504 (2012) [arXiv:1206.3968 [hep-ph]].

[35] T. Huang, T. Zhong and X. -G. Wu, Phys. Rev. D 88, 034013 (2013) [arXiv:1305.7391 [hep$\mathrm{ph}]$.

[36] P. Kroll, Eur. Phys. J. C 71, 1623 (2011) [arXiv:1012.3542 [hep-ph]].

[37] R. Delbourgo and M. D. Scadron, J. Phys. G 5, 1621 (1979).

[38] M. B. Parappilly, P. O. Bowman, U. M. Heller, D. B. Leinweber, A. G. Williams and J. B. Zhang, Phys. Rev. D 73, 054504 (2006).

[39] P. O. Bowman, U. M. Heller, D. B. Leinweber and A. G. Williams, Nucl. Phys. Proc. Suppl. 119, 323 (2003); P. O. Bowman, U. M. Heller, and A. G. Williams, Phys. Rev. D 66, 014505 (2002). 
[40] R. D. Bowler and M. C. Birse, Nucl. Phys. A 582, 655 (1995) [arXiv:hep-ph/9407336]; R. S. Plant and M. C. Birse, Nucl. Phys. A 628, 607 (1998) [arXiv:hep-ph/9705372].

[41] A. Scarpettini, D. Gomez Dumm and N. N. Scoccola, Phys. Rev. D 69, 114018 (2004) [arXiv:hep-ph/0311030].

[42] D. Gomez Dumm, A. G. Grunfeld and N. N. Scoccola, Phys. Rev. D 74, 054026 (2006) [arXiv:hep-ph/0607023].

[43] B. Golli, W. Broniowski and G. Ripka, Phys. Lett. B 437, 24 (1998) [arXiv:hep-ph/9807261]; W. Broniowski, B. Golli and G. Ripka, Nucl. Phys. A 703, 667 (2002) [arXiv:hep-ph/0107139].

[44] A. H. Rezaeian, N. R. Walet and M. C. Birse, Phys. Rev. C 70, 065203 (2004) [arXiv:hepph/0408233]; A. H. Rezaeian and H. J. Pirner, Nucl. Phys. A 769, 35 (2006) [arXiv:nuclth/0510041].

[45] S. Noguera, Int. J. Mod. Phys. E 16, 97 (2007) [arXiv:hep-ph/0806.0818].

[46] S. Noguera and V. Vento, Eur. Phys. J. A 28, 227 (2006) [arXiv:hep-ph/0505102].

[47] S. Noguera and N. N. Scoccola, Phys. Rev. D 78, 114002 (2008) [arXiv:hep-ph/0806.0818].

[48] D. Gomez Dumm, S. Noguera and N. N. Scoccola, Phys. Lett. B 698, 236 (2011) [arXiv:1011.6403 [hep-ph]]; Phys. Rev. D 86, 074020 (2012) [arXiv:1205.2730 [hep-ph]].

[49] F. del Aguila and M. K. Chase, Nucl. Phys. B 193, 517 (1981).

[50] E. Braaten, Phys. Rev. D 28, 524 (1983).

[51] G. F. Sterman and P. Stoler, Ann. Rev. Nucl. Part. Sci. 47, 193 (1997) [hep-ph/9708370].

[52] E. M. Aitala et al. [E791 Collaboration], Phys. Rev. Lett. 86, 4768 (2001) [hep-ex/0010043].

[53] A. R. Zhitnitsky, Phys. Lett. B 329, 493 (1994) [hep-ph/9401278].

[54] A. Gonzalez-Arroyo, C. Lopez and F. J. Yndurain, Nucl. Phys. B 153, 161 (1979).

[55] E. G. Floratos, D. A. Ross and C. T. Sachrajda, Nucl. Phys. B 129, 66 (1977) [Erratum-ibid. B 139, $545(1978)]$. 